\title{
Review: a pooled long term persistence rate of $40 \%$ for childhood $O C D$ is lower than previously expected
}

Stewart SE, Geller DA, Jenike M, et al. Long-term outcome of pediatric obsessive compulsive disorder: a meta analysis and qualitative review of the literature. Acta Psychiatr Scand 2004;110:4-13.

What is the long term prognosis for children and adolescents with obsessive compulsive disorder?

METHODS

Design: Systematic review with meta-analysis

Data sources: MEDLINE and PsycLIT database searches; search date not reported.

Study selection and analysis: Long term ( $>1$ year) follow up studies of obsessive compulsive disorder originating in childhood or adolescence. Predictors and persistence of OCD were metaanalysed using the DerSimonian and Laird model and effect sizes were calculated. Meta regression analysis was used to correlate persistence with age of onset, age of assessment, OCD duration, sex, treatment centre, and year. Follow up periods among included studies ranged from 1 to 15.6 years (mean 5.7 years); mean onset age was 10.4 years (range $7.7-12.5$ years) and mean study entry age was 13.3 (11.8-15 years).

Find Outcomes: Percentage OCD at follow up (full or subthreshold).

\section{MAIN RESULTS}

Sixteen studies in 22 reports $(n=521)$ met inclusion criteria. At endpoint, full OCD had a persistence rate of $41 \%$ (95\% CI $32 \%$ to $51 \%)$; combined full and subthreshold OCD had a persistence rate of $60 \%$ (95\% CI 0.46 to 0.74 ). Predictors of persistence were early onset $(\mathrm{p}=0.001)$, increased OCD duration $(\mathrm{p}=0.027)$, and inpatient versus outpatient status $(\mathrm{p}=0.003)$. Sex, age of assessment, length of follow up period, and year did not significantly predict persistence. Five studies reported high levels of problems with peers, isolation, and problems with employment, although educational attainment did not differ from peers.

For correspondence: D A Geller, Pediatric Psychopharmacology Research Program, and 185 Alewife Brook Parkway, Suite 2000, Cambridge, MA 02138, USA; dageller@partners.org

Sources of funding: Canadian Institutes of Health Research, University of Ottawa International, Obsessive Compulsive Foundation award, NMIH.

\section{CONCLUSIONS}

Persistence of paediatric OCD may be lower than previously thought.

\section{Commentary}

decade ago, little was known about the evidence-based treatment, long term treatment, or non-treatment of paediatric obsessive compulsive disorder (OCD). That sufficient reports have accumulated to permit the meta-analysis by Stewart and colleagues ${ }^{1}$ is in itself noteworthy. Unfortunately, Stewart et al were unable to examine the moderating effect of treatment and, specifically, of medical management with selective serotonin reuptake inhibitors (SSRI) or with cognitive behavioural therapy (CBT), therefore sharply limiting the utility of the results. Given that most patients probably did not receive expert CBT and were not consistently medicated, persistence of partial or full OCD is not surprising. New research indicates that combined treatment with an SSRI and a widely available CBT protocol ${ }^{2}$ induces clinical remission in over $50 \%$ of children and adolescents with OCD after 12 weeks of treatment. ${ }^{3}$ Hence it is reasonable, based on existing evidence, that patients should start treatment with CBT or the combination of CBT with an SSRI. As implied in this meta-analysis, medication monotherapy, although helpful, is not as satisfactory as initial treatment of OCD in children and adolescents. As CBT is not widely available, the key to improving the public health of children and adolescents with $O C D$ is wider availability of OCD specific, developmentally sensitive, cognitive behavioural psychotherapy. It remains to be seen, however, whether future reports will show that evidence-based treatment has an impact on long term outcomes.

John S March, MD, MPH

Professor and Chief, Child and Adolescent Psychiatry, Department of Psychiatry and Behavioral Sciences, Duke Child and Family Study Center, North Carolina, USA

1 Stewart SE, Geller DA, Jenike M, et al. Long-term outcome of pediatric obsessive-compulsive disorder: a meta-analysis and qualitative review of the literature. Acta Psychiatr Scand 2004;1 10:4-13.

2 March J, Mulle K. OCD in children and adolescents: a cognitive-behavioral treatment manual. New York: Guilford Press, 1998.

3 Pediatric Obsessive Compulsive Disorder Treatment Study Team. Cognitivebehavior therapy, sertraline, and their combination for children and adolescents with obsessive-compulsive disorder: the Pediatric OCD Treatment Study (POTS) randomized controlled trial. JAMA 2004:292:1969-76. 